\title{
BORN-JORDAN PSEUDODIFFERENTIAL OPERATORS WITH SYMBOLS IN THE SHUBIN CLASSES
}

\author{
ELENA CORDERO, MAURICE DE GOSSON, AND FABIO NICOLA
}

\begin{abstract}
We apply Shubin's theory of global symbol classes $\Gamma_{\rho}^{m}$ to the Born-Jordan pseudodifferential calculus we have previously developed. This approach has many conceptual advantages and makes the relationship between the conflicting Born-Jordan and Weyl quantization methods much more limpid. We give, in particular, precise asymptotic expansions of symbols allowing us to pass from Born-Jordan quantization to Weyl quantization and vice versa. In addition we state and prove some regularity and global hypoellipticity results.
\end{abstract}

\section{INTRODUCTION}

The Born-Jordan quantization rules 4, 5, 7, have recently been rediscovered in mathematics and have quickly become a very active area of research under the impetus of scientists working in signal theory and time-frequency analysis [1, 8, 11. It has been realized not only that the associated phase space picture has many advantages compared with the usual Weyl-Wigner picture (it allows a strong damping of unwanted interference patterns [1,10,30]), but also, as shown by de Gosson [18 20], that there is strong evidence that Born-Jordan quantization might very well be the correct quantization method in quantum physics. Independently of these potential applications, the Born-Jordan pseudodifferential calculus has many interesting and difficult features (some of them, such as non-injectivity [9, being even quite surprising) and deserve close attention. The involved mathematics is less straightforward than that of the usual Weyl formalism; for instance Born-Jordan pseudodifferential calculus is not fully covariant under linear symplectic transformations [16, which makes the study of the symmetries of the operators much less straightforward than in the Weyl case.

In the present paper we set out to study the pseudodifferential calculus associated with Born-Jordan quantization in the framework of Shubin's 28, global symbol classes. These results complement and extend those obtained by the authors in 9 .

To be precise, in the Weyl quantization scheme, to any observable (symbol) $a(z)$, $z \in \mathbb{R}^{2 n}$, defined as a function or (temperate) distribution in phase space, there is associated the Weyl operator

$$
\widehat{A}_{\mathrm{W}}=\left(\frac{1}{2 \pi \hbar}\right)^{n} \int a_{\sigma}(z) \widehat{T}(z) d^{2 n} z,
$$

Received by the editors March 10, 2016, and, in revised form, January 4, 2017.

2010 Mathematics Subject Classification. Primary 35S05; Secondary 46L65.

Key words and phrases. Born-Jordan quantization, pseudodifferential operators, Shubin classes, Weyl quantization.

The second author was supported by the Austrian Research Foundation (FWF) grant P27773N23.

(C) 2017 by the authors under Creative Commons Attribution-Noncommercial 3.0 License (CC BY NC 3.0) 
where $a_{\sigma}=F_{\sigma} a$ is the symplectic Fourier transform of $a$ and $\widehat{T}\left(z_{0}\right)$ is the Heisenberg operator given by

$$
\widehat{T}\left(z_{0}\right) \psi(x)=e^{\frac{i}{\hbar}\left(p_{0} x-\frac{1}{2} p_{0} x_{0}\right)} \psi\left(x-x_{0}\right) .
$$

This is simply a phase space shift and, as a consequence of the Schwartz kernel theorem, every continuous linear operator $\mathcal{S}\left(\mathbb{R}^{n}\right) \rightarrow \mathcal{S}^{\prime}\left(\mathbb{R}^{n}\right)$ can be written in a unique way as a Weyl operator for a suitable symbol $a \in \mathcal{S}^{\prime}\left(\mathbb{R}^{2 n}\right)$; namely, it is a superposition of phase space shifts. In this functional framework the Weyl correspondence between observables and operators is therefore one-to-one.

The Born-Jordan quantization of a symbol $a(z)$ is instead defined as

$$
\widehat{A}_{\mathrm{BJ}}=\left(\frac{1}{2 \pi \hbar}\right)^{n} \int a_{\sigma}(z) \operatorname{sinc}\left(\frac{p x}{2 \hbar}\right) \widehat{T}(z) d^{2 n} z
$$

with $z=(x, p)$ and $p x=p \cdot x$. The presence of the function sinc $\left(\frac{p x}{2 \hbar}\right)$ and in particular its zeros make the corresponding quantization problem much more subtle. It was proved in $\left[9\right.$ that every linear continuous operator $\mathcal{S}\left(\mathbb{R}^{n}\right) \rightarrow \mathcal{S}^{\prime}\left(\mathbb{R}^{n}\right)$ can still be written in Born-Jordan form, but the representation is no longer unique. The Born-Jordan correspondence is anyway still surjective.

In this paper we continue this investigation by focusing on a particulary relevant subclass of smooth symbols satisfying good growth conditions at infinity, namely Shubin's classes [28]. Roughly speaking the main result reads as follows. Within such symbol classes the Weyl symbol $a_{\mathrm{W}}$ and the corresponding Born-Jordan symbol $a_{\mathrm{BJ}}$ are related by the following explicit asymptotic expansions:

$$
a_{\mathrm{W}}(x, p) \sim \sum_{\substack{\alpha \in \mathbb{N}^{n} \\|\alpha| \text { even }}} \frac{1}{\alpha !(|\alpha|+1)}\left(\frac{i \hbar}{2}\right)^{|\alpha|} \partial_{x}^{\alpha} \partial_{p}^{\alpha} a_{\mathrm{BJ}}(x, p)
$$

and

$$
a_{\mathrm{BJ}}(x, p) \sim \sum_{\substack{\alpha \in \mathbb{N}^{n} \\|\alpha| \text { even }}} \frac{c_{\alpha}}{\alpha !}\left(\frac{i \hbar}{2}\right)^{|\alpha|} \partial_{x}^{\alpha} \partial_{p}^{\alpha} a_{\mathrm{W}}(x, p)
$$

for suitable coefficients $c_{\alpha}$ (see (4.10) below).

These expansions seem remarkable, because at present there is no an exact and explicit formula for the Born-Jordan symbol corresponding to a given Weyl operator, although the existence of such a symbol was proved in [9]. Indeed, the situation seems definitely similar to what happens in the division problem of temperate distributions by a (not identically zero) polynomial $P$ : the map $f \mapsto P f$ from $\mathcal{S}^{\prime}\left(\mathbb{R}^{n}\right)$ into itself is onto but in general a linear continuous right inverse does not exist 3,25 .

We will systematically use properties of the global pseudodifferential calculus whose study was initiated by Shubin, after related work by Beals, Berezin, Kumanogo, Rabinovič, and others (see the bibliography in [28]). This calculus plays an important role in quantum mechanics since the position and momentum variables are placed on an equal footing in the estimates defining the symbol classes. We have found this approach particularly well adapted to investigate asymptotic expansions such as those for $a_{\mathrm{W}}$ and $a_{\mathrm{BJ}}$.

Natural related topics that we have not included in this work are the spectral theory of Born-Jordan operators, in which the notion of global hypoellipticity plays a crucial role, and the anti-Wick version of these operators (the latter might lead 
to some new insights). Finally, we have not discussed at all the Wigner-Moyal formalism associated with the Born-Jordan question; for the latter we refer to [1, 10, 18.

In short, the paper is organized as follows. In Section 2 we review the definition of the Born-Jordan pseudodifferential operators. Section 3 is devoted to Shubin's symbol classes. In Section 4 we prove the above relationships between Weyl and Born-Jordan symbols. Finally Section 5 is devoted to applications to the global regularity problem.

Notation. We denote by $\sigma$ the standard symplectic form $\sum_{j=1}^{n} d p_{j} \wedge d x_{j}$ on the phase space $\mathbb{R}^{2 n} \equiv \mathbb{R}^{n} \times \mathbb{R}^{n}$; the phase space variable is written $z=(x, p)$. Equivalently, $\sigma\left(z, z^{\prime}\right)=J z \cdot z^{\prime}$ where $J=\left(\begin{array}{cc}0 & I \\ -I & 0\end{array}\right)$. We will denote by $\widehat{x}_{j}$ the operator of multiplication by $x_{j}$ and set $\widehat{p}_{j}=-i \hbar \partial / \partial x_{j}$. These operators satisfy Born's canonical commutation relations $\left[\widehat{x}_{j}, \widehat{p}_{j}\right]=i \hbar$, where $\hbar$ is a positive parameter such that $0<\hbar \leq 1$.

\section{BORN-JORDAN PSEUDODIFFERENTIAL OPERATORS}

In this section we review the recent advances in the theory of Born-Jordan quantization; for proofs and details we refer to Cordero et al. [9] and de Gosson [16, 18, 19].

2.1. The Born-Jordan quantization rules. Following Heisenberg's insightful work on "matrix mechanics" Born and Jordan [4,5] proposed the quantization rule

$$
p^{s} x^{r} \stackrel{\mathrm{BJ}}{\longrightarrow} \frac{1}{s+1} \sum_{\ell=0}^{s} \widehat{p}^{s-\ell} \widehat{x}^{r} \widehat{p}^{\ell}
$$

for monomials. Their rule conflicts with Weyl's 31] quantization rule, leading to

$$
p^{s} x^{r} \stackrel{\mathrm{W}}{\longrightarrow} \frac{1}{2^{s}} \sum_{\ell=0}^{s}\left(\begin{array}{l}
s \\
\ell
\end{array}\right) \widehat{p}^{s-\ell} \widehat{x}^{r} \widehat{p}^{\ell}
$$

(McCoy rule [27]) as soon as $r \geq 2$ and $s \geq 2$. The following observation is crucial: both quantizations are obtained from Shubin's $\tau$-rule

$$
p^{s} x^{r} \stackrel{\tau}{\longrightarrow} \sum_{\ell=0}^{s}\left(\begin{array}{l}
s \\
\ell
\end{array}\right)(1-\tau)^{\ell} \tau^{s-\ell} \widehat{p}^{s-\ell} \widehat{x}^{r} \widehat{p}^{\ell}
$$

but by very different means. In fact, the Weyl rule (2.2) is directly obtained by choosing $\tau=\frac{1}{2}$ while Born and Jordan's rule (2.1) is obtained by averaging the right-hand side of (2.3) with respect to $\tau$ over the interval $[0,1]$ (de Gosson and Luef [21, de Gosson [16, 18]).

On the operator level, the Weyl operator $\widehat{A}_{\mathrm{W}}=\mathrm{Op}_{\mathrm{W}}(a)$ is given by the familiar formula due to Weyl himself 31]

$$
\widehat{A}_{\mathrm{W}}=\left(\frac{1}{2 \pi \hbar}\right)^{n} \int a_{\sigma}(z) \widehat{T}(z) d^{2 n} z,
$$

where $a_{\sigma}=F_{\sigma} a$ is the symplectic Fourier transform

$$
a_{\sigma}(z)=\left(\frac{1}{2 \pi \hbar}\right)^{n} \int e^{-\frac{i}{\hbar} \sigma\left(z, z^{\prime}\right)} a\left(z^{\prime}\right) d^{2 n} z^{\prime}
$$


and $\widehat{T}\left(z_{0}\right)=e^{-\frac{i}{\hbar} \sigma\left(\hat{z}, z_{0}\right)}$ is the Heisenberg operator; recall [14,26] that the action of $\widehat{T}\left(z_{0}\right)$ on a function or distribution $\psi$ is explicitly given by

$$
\widehat{T}\left(z_{0}\right) \psi(x)=e^{\frac{i}{\hbar}\left(p_{0} x-\frac{1}{2} p_{0} x_{0}\right)} \psi\left(x-x_{0}\right) .
$$

Let us underline that the parameter $\hbar \in(0,1]$ is fixed in our context. Here we are not interested in the semiclassical analysis, i.e., the asymptotic as $\hbar \rightarrow 0$.

Using Plancherel's identity, formula (2.4) can be rewritten

$$
\widehat{A}_{\mathrm{W}}=\left(\frac{1}{\pi \hbar}\right)^{n} \int a(z) \widehat{\Pi}(z) d^{2 n} z,
$$

where

$$
\widehat{\Pi}(z)=\widehat{T}(z) \widehat{\Pi} \widehat{T}(z)^{-1}
$$

is the Grossmann-Royer reflection operator (where $\widehat{\Pi} \psi(x)=\psi(-x)$ ). One verifies that under suitable convergence conditions (for instance $a \in \mathcal{S}\left(\mathbb{R}^{2 n}\right.$ ) and $\left.\psi \in L^{1}\left(\mathbb{R}^{n}\right)\right)$ one recovers the more familiar "midpoint formula"

$$
\widehat{A}_{\mathrm{W}} \psi(x)=\left(\frac{1}{2 \pi \hbar}\right)^{n} \iint e^{\frac{i}{\hbar} p(x-y)} a\left(\frac{1}{2}(x+y), p\right) \psi(y) d^{n} y d^{n} p
$$

common in the theory of pseudodifferential operators; we will use this notation as a formal tool for the sake of clarity (keeping in mind that it can be given a rigorous meaning by (2.7D). The easiest way to define Shubin's $\tau$-operator $\widehat{A}_{\tau}=\mathrm{Op}_{\tau}(a)$ is to use the formula above as a starting point, and to replace the midpoint $\frac{1}{2}(x+y)$ with $(1-\tau) x+\tau y$ which leads to

$$
\widehat{A}_{\tau} \psi(x)=\left(\frac{1}{2 \pi \hbar}\right)^{n} \iint e^{\frac{i}{\hbar} p(x-y)} a((1-\tau) x+\tau y, p) \psi(y) d^{n} y d^{n} p .
$$

As in the monomial case, the Born-Jordan operator $\widehat{A}_{\mathrm{BJ}}=\mathrm{Op}_{\mathrm{BJ}}(a)$ is obtained by averaging (2.10) over $[0,1]$ :

$$
\widehat{A}_{\mathrm{BJ}}=\int_{0}^{1} \widehat{A}_{\tau} d \tau
$$

2.2. Harmonic representation of Born-Jordan operators. The following result gives an explicit expression of the Weyl symbol of a Born-Jordan operator with arbitrary symbol (see [1,9]).

Proposition 2.1. Let $a \in \mathcal{S}^{\prime}\left(\mathbb{R}^{2 n}\right)$.

(i) The operator $\widehat{A}_{\mathrm{BJ}}=\mathrm{Op}_{\mathrm{BJ}}(a)$ is the Weyl operator $\mathrm{Op}_{\mathrm{W}}(b)$, where

$$
b(x, p)=\left(\frac{1}{2 \pi \hbar}\right)^{n}(a * \theta)(x, p) ;
$$

here $\theta \in S^{\prime}\left(\mathbb{R}^{2 n}\right)$ is the distribution whose (symplectic) Fourier transform is

$$
\theta_{\sigma}(x, p)=\operatorname{sinc}\left(\frac{p x}{2 \hbar}\right) .
$$

(ii) The restriction of $\widehat{A}_{\mathrm{BJ}}$ to monomials $p_{j}^{s} x_{j}^{r}$ is given by the Born-Jordan rule (2.1).

Recall that the function $\operatorname{sinc}$ is defined by $\operatorname{sinc} u=\sin u / u$ for $u \neq 0$ and $\operatorname{sinc} 0=1$. 
It follows from (2.4) and the convolution formula $F_{\sigma}(a * \theta)=(2 \pi \hbar)^{n} a_{\sigma} \theta_{\sigma}$ that $\widehat{A}_{\mathrm{BJ}}$ is alternatively given by

$$
\widehat{A}_{\mathrm{BJ}}=\left(\frac{1}{2 \pi \hbar}\right)^{n} \int a_{\sigma}(z) \operatorname{sinc}\left(\frac{p x}{2 \hbar}\right) \widehat{T}(z) d^{2 n} z
$$

(cf. formula (2.4) for Weyl operators).

\section{SyMbOL CLASSES}

In what follows we use the notation $\langle u\rangle=\sqrt{1+|u|^{2}}$ for $u \in \mathbb{R}^{m}$. For instance, if $z=(x, p) \in \mathbb{R}^{2 n}$, then

$$
\langle z\rangle=\sqrt{1+|z|^{2}}=\sqrt{1+|x|^{2}+|p|^{2}} .
$$

We assume that the reader is familiar with multi-index notation: if $u=\left(u_{1}, \ldots, u_{m}\right)$ $\in \mathbb{R}^{m}$ and $\alpha=\left(\alpha_{1}, \ldots, \alpha_{m}\right) \in \mathbb{N}^{m}$ we write $u^{\alpha}=u_{1}^{\alpha_{1}} \cdots u_{m}^{\alpha_{m}} ;$ similarly $\partial_{u}^{\alpha}=$ $\partial_{u_{1}}^{\alpha_{1}} \cdots \partial_{u_{m}}^{\alpha_{m}}$. By definition $|\alpha|=\alpha_{1}+\cdots+\alpha_{m}$ and $\alpha !=\alpha_{1} ! \cdots \alpha_{m} !$

3.1. The Shubin symbol class $\Gamma_{\rho}^{m}$. We begin by giving the following definition (Shubin [28, Definition 23.1).

Definition 3.1. Let $m \in \mathbb{R}$ and $0<\rho \leq 1$. The symbol class $\Gamma_{\rho}^{m}\left(\mathbb{R}^{2 n}\right)$ consists of all complex functions $a \in C^{\infty}\left(\mathbb{R}^{2 n}\right)$ such that for every $\alpha \in \mathbb{N}^{2 n}$ there exists a constant $C_{\alpha} \geq 0$ with

$$
\left|\partial_{z}^{\alpha} a(z)\right| \leq C_{\alpha}\langle z\rangle^{m-\rho|\alpha|} \text { for } z \in \mathbb{R}^{2 n} .
$$

It immediately follows from this definition that if $a \in \Gamma_{\rho}^{m}\left(\mathbb{R}^{2 n}\right)$ and $\alpha \in \mathbb{N}^{2 n}$, then $\partial_{z}^{\alpha} a \in \Gamma_{\rho}^{m-\rho|\alpha|}\left(\mathbb{R}^{2 n}\right)$; using Leibniz's rule for the derivative of products of functions one easily checks that

$$
a \in \Gamma_{\rho}^{m}\left(\mathbb{R}^{2 n}\right) \text { and } b \in \Gamma_{\rho}^{m^{\prime}}\left(\mathbb{R}^{2 n}\right) \Longrightarrow a b \in \Gamma_{\rho}^{m+m^{\prime}}\left(\mathbb{R}^{2 n}\right) .
$$

The class $\Gamma_{\rho}^{m}\left(\mathbb{R}^{2 n}\right)$ is a complex vector space for the usual operations of addition and multiplication by complex numbers, and we have

$$
\Gamma_{\rho}^{-\infty}\left(\mathbb{R}^{2 n}\right)=\bigcap_{m \in \mathbb{R}} \Gamma_{\rho}^{m}\left(\mathbb{R}^{2 n}\right)=\mathcal{S}\left(\mathbb{R}^{2 n}\right) .
$$

The reduced harmonic oscillator Hamiltonian $H(z)=\frac{1}{2}\left(|x|^{2}+|p|^{2}\right)$ obviously belongs to $\Gamma_{1}^{2}\left(\mathbb{R}^{2 n}\right)$, and so does

$$
H(z)=\sum_{j=1}^{n} \frac{1}{2 m_{j}}\left(p_{j}^{2}+m_{j}^{2} \omega_{j}^{2} x_{j}^{2}\right) ;
$$

in fact, any polynomial function in $z$ of degree $m$ is in $\Gamma_{1}^{m}\left(\mathbb{R}^{2 n}\right)$. In particular every Hamiltonian function of the type

$$
H(z)=\sum_{j=1}^{n} \frac{1}{2 m_{j}} p_{j}^{2}+V(x)
$$

belongs to some class $\Gamma_{1}^{m}\left(\mathbb{R}^{2 n}\right)$ if the potential function $V(x)$ is a polynomial of degree $m \geq 2$.

The following lemma shows that the symbol classes $\Gamma_{\rho}^{m}\left(\mathbb{R}^{2 n}\right)$ are invariant under linear automorphisms of phase space (this property does not hold for the usual 
Hörmander classes $S_{\rho, \delta}^{m}\left(\mathbb{R}^{n}\right)[24$, whose elements are characterized by growth properties in only the variable $p)$. Let us denote by $G L(2 n, \mathbb{R})$ the space of $2 n \times 2 n$ invertible real matrices. Then

Lemma 3.2. Let $a \in \Gamma_{\rho}^{m}\left(\mathbb{R}^{2 n}\right)$ and $M \in G L(2 n, \mathbb{R})$. We have $a(M \cdot) \in \Gamma_{\rho}^{m}\left(\mathbb{R}^{2 n}\right)$.

Proof. The result is shown in greater generality in [28, p. 177]. This special case simply follows by the fact

$$
C^{-1}|z| \leq|M z| \leq C|z|
$$

for a suitable $C>0$.

3.2. Asymptotic expansions of symbols. Let us recall the notion of asymptotic expansion of a symbol $a \in \Gamma_{\rho}^{m}\left(\mathbb{R}^{2 n}\right)$ (cf. [28], Definition 23.2).

Definition 3.3. Let $\left(a_{j}\right)_{j}$ be a sequence of symbols $a_{j} \in \Gamma_{\rho}^{m_{j}}\left(\mathbb{R}^{2 n}\right)$ such that $\lim _{j \rightarrow+\infty} m_{j} \rightarrow-\infty$. Let $a \in C^{\infty}\left(\mathbb{R}^{2 n}\right)$. If for every integer $r \geq 2$ we have

$$
a-\sum_{j=1}^{r-1} a_{j} \in \Gamma_{\rho}^{\bar{m}_{r}}\left(\mathbb{R}^{2 n}\right),
$$

where $\bar{m}_{r}=\max _{j \geq r} m_{j}$, we will write $a \sim \sum_{j=1}^{\infty} a_{j}$ and call this relation an asymptotic expansion of the symbol $a$.

The interest of the asymptotic expansion comes from the fact that every sequence of symbols $\left(a_{j}\right)_{j}$ with $a_{j} \in \Gamma_{\rho}^{m_{j}}\left(\mathbb{R}^{2 n}\right)$, the degrees $m_{j}$ being strictly decreasing and such that $m_{j} \rightarrow-\infty$, determines a symbol in some $\Gamma_{\rho}^{m}\left(\mathbb{R}^{2 n}\right)$, that symbol being unique up to an element of $\mathcal{S}\left(\mathbb{R}^{2 n}\right)$ :

Proposition 3.4. Let $\left(a_{j}\right)_{j}$ be a sequence of symbols $a_{j} \in \Gamma_{\rho}^{m_{j}}\left(\mathbb{R}^{2 n}\right)$ such that $m_{j}>m_{j+1}$ and $\lim _{j \rightarrow+\infty} m_{j} \rightarrow-\infty$. Then:

(i) There exists a function a such that $a \sim \sum_{j=1}^{\infty} a_{j}$.

(ii) If another function $a^{\prime}$ is such that $a^{\prime} \sim \sum_{j=1}^{\infty} a_{j}$, then $a-a^{\prime} \in \mathcal{S}\left(\mathbb{R}^{2 n}\right)$.

(See Shubin 28, Proposition 23.1.) Note that property (ii) immediately follows from (3.3).

3.3. The amplitude classes $\Pi_{\rho}^{m}$. We will need for technical reasons an extension of the Shubin classes $\Gamma_{\rho}^{m}\left(\mathbb{R}^{2 n}\right)$ defined above. Since Born-Jordan operators are obtained by averaging Shubin's $\tau$-operators

$$
\widehat{A}_{\tau} \psi(x)=\left(\frac{1}{2 \pi \hbar}\right)^{n} \iint e^{\frac{i}{\hbar} p(x-y)} a((1-\tau) x+\tau y, p) \psi(y) d^{n} y d^{n} p
$$

over $\tau \in[0,1]$, we are led to consider pseudodifferential operators of the type

$$
\widehat{A} \psi(x)=\left(\frac{1}{2 \pi \hbar}\right)^{n} \iint e^{\frac{i}{\hbar} p(x-y)} b(x, y, p) \psi(y) d^{n} y d^{n} p,
$$

where the function

$$
b(x, y, p)=\int_{0}^{1} a((1-\tau) x+\tau y, p) d \tau
$$

is called the amplitude and is defined not on $\mathbb{R}^{2 n} \equiv \mathbb{R}_{x}^{n} \times \mathbb{R}_{p}^{n}$ but rather on $\mathbb{R}^{3 n} \equiv$ $\mathbb{R}_{x}^{n} \times \mathbb{R}_{y}^{n} \times \mathbb{R}_{p}^{n}$. It therefore makes sense to define an amplitude class generalizing $\Gamma_{\rho}^{m}\left(\mathbb{R}^{2 n}\right)$ by allowing a dependence on the three sets of variables $x, y$, and $p$ (cf. 28, Definition 23.3). 
Definition 3.5. Let $m \in \mathbb{R}$. The symbol (or amplitude) class $\Pi_{\rho}^{m}\left(\mathbb{R}^{3 n}\right)$ consists of all functions $a \in C^{\infty}\left(\mathbb{R}^{3 n}\right)$ that for some $m^{\prime} \in \mathbb{R}$ satisfy

$$
\left|\partial_{p}^{\alpha} \partial_{x}^{\beta} \partial_{y}^{\gamma} a(x, y, p)\right| \leq C_{\alpha \beta \gamma}\langle u\rangle^{m-\rho|\alpha+\beta+\gamma|}\langle x-y\rangle^{m^{\prime}+\rho|\alpha+\beta+\gamma|}
$$

for every $(\alpha, \beta, \gamma) \in \mathbb{N}^{3 n}$, where $C_{\alpha \beta \gamma} \geq 0$ and $u=(x, y, p)$.

It turns out that an operator (3.5) with amplitude $b \in \Pi_{\rho}^{m}\left(\mathbb{R}^{3 n}\right)$ is a Shubin $\tau$-pseudodifferential operator with symbol in $\Gamma_{\rho}^{m}\left(\mathbb{R}^{2 n}\right)$ - and this for every value of the parameter $\tau$ :

Proposition 3.6. Let $\tau$ be an arbitrary real number.

(i) Every pseudodifferential operator $\widehat{A}$ of the type (3.5) with amplitude $b \in$ $\Pi_{\rho}^{m}\left(\mathbb{R}^{3 n}\right)$ can be uniquely written in the form $\widehat{A}=\mathrm{Op}_{\tau}\left(a_{\tau}\right)$ for some symbol $a_{\tau} \in \Gamma_{\rho}^{m}\left(\mathbb{R}^{2 n}\right)$, that is,

$$
\widehat{A} \psi(x)=\left(\frac{1}{2 \pi \hbar}\right)^{n} \iint e^{\frac{i}{\hbar} p(x-y)} a_{\tau}((1-\tau) x+\tau y, p) \psi(y) d^{n} y d^{n} p ;
$$

the symbol $a_{\tau}$ has the asymptotic expansion

$$
\left.a_{\tau}(x, p) \sim \sum_{\beta, \gamma} \frac{1}{\beta ! \gamma !} \tau^{|\beta|}(1-\tau)^{|\gamma|} \partial_{p}^{\beta+\gamma}\left(i \hbar \partial_{x}\right)^{\beta}\left(-i \hbar \partial_{y}\right)^{\gamma} b(x, y, p)\right|_{y=x}
$$

(ii) In particular, choosing $\tau=\frac{1}{2}$, there exists $a_{\mathrm{W}} \in \Gamma_{\rho}^{m}\left(\mathbb{R}^{2 n}\right)$ such that $\widehat{A}=$ $\mathrm{Op}_{\mathrm{W}}\left(a_{\mathrm{W}}\right)$.

Proof. See Shubin [28, Theorem 23.2, for the case $\hbar=1$ and de Gosson [15], Section 14.2.2.

We have in addition an asymptotic formula allowing us to pass from one $\tau$ symbol to another when $\widehat{A}$ is given by (3.7): if $\widehat{A}=\mathrm{Op}_{\tau}\left(a_{\tau}\right)=\mathrm{Op}_{\tau^{\prime}}\left(a_{\tau^{\prime}}\right)$ with $a_{\tau}, a_{\tau^{\prime}} \in \Pi_{\rho}^{m}\left(\mathbb{R}^{3 n}\right)$, then

$$
a_{\tau}(x, p) \sim \sum_{\alpha \geq 0} \frac{i^{-|\alpha|}}{\alpha !}\left(\tau^{\prime}-\tau\right)^{|\alpha|} \partial_{p}^{\alpha} \partial_{x}^{\alpha} a_{\tau^{\prime}}(x, p)
$$

([28, Theorem 23.3).

3.4. Elementary properties. The class of all operators (3.5) with $b \in \Pi_{\rho}^{m}\left(\mathbb{R}^{3 n}\right)$ is denoted by $G_{\rho}^{m}\left(\mathbb{R}^{n}\right)$ (cf. [28, Definition 23.4); $G^{-\infty}\left(\mathbb{R}^{n}\right)=\bigcap_{m \in \mathbb{R}} G_{\rho}^{m}\left(\mathbb{R}^{n}\right)$ consists of all operators $\mathcal{S}\left(\mathbb{R}^{n}\right) \longrightarrow \mathcal{S}\left(\mathbb{R}^{n}\right)$ with distributional kernel $K \in \mathcal{S}\left(\mathbb{R}^{n} \times \mathbb{R}^{n}\right)$. It is useful to make the following remark: in the standard theory of pseudodifferential operators (notably in its applications to partial differential operators) it is customary to use operators

$$
\widehat{A} \psi(x)=\left(\frac{1}{2 \pi}\right)^{n} \iint e^{i(x-y) \xi} a(x, y, \xi) \psi(y) d^{n} y d^{n} \xi
$$

which correspond, replacing $p$ with $\xi$ to the choice $\hbar=1$ in the expression (3.5). It is in fact easy to toggle between the expression above and its $\hbar$-dependent version; one just replaces $a(x, y, \xi)$ with $a(x, y, p)$ and $\xi$ with $p / \hbar$ so that $d \xi=\hbar^{-n} d p$. However, when doing this, one must be careful to check that the amplitudes $a(x, y, \xi)$ and $a(x, y, \hbar \xi)$ belong to the same symbol class. That this is indeed always the case 
when one deals with Shubin classes is clear from Lemma 3.2. The following situation is important in our context; consider the $\hbar=1$ Weyl operator

$$
\widehat{A} \psi(x)=\left(\frac{1}{2 \pi}\right)^{n} \iint e^{i(x-y) \xi} a\left(\frac{1}{2}(x+y), \xi\right) \psi(y) d^{n} y d^{n} \xi .
$$

Denoting by $\widehat{A}^{(\hbar)}$ the corresponding operator 3.5 in order to make the $\hbar$ dependence clear, that is,

$$
\widehat{A}^{(\hbar)} \psi(x)=\left(\frac{1}{2 \pi \hbar}\right)^{n} \iint e^{\frac{i}{\hbar} p(x-y)} a\left(\frac{1}{2}(x+y), p\right) \psi(y) d^{n} y d^{n} p,
$$

we have $\widehat{A}^{(\hbar)}=\widehat{M}_{\hbar}^{-1} \widehat{B} \widehat{M}_{\hbar}$ where $\widehat{B}$ is the operator (3.10) with symbol $b(x, p)=$ $a\left(\hbar^{1 / 2} x, \hbar^{1 / 2} p\right)$ and $\widehat{M}_{\hbar}$ is the unitary scaling operator defined by $\widehat{M}_{\hbar} \psi(x)=$ $\hbar^{n / 4} \psi\left(\hbar^{1 / 2}\right)$.

Using the symbol estimates (3.1) it is straightforward to show that every operator $\widehat{A} \in G_{\rho}^{m}\left(\mathbb{R}^{n}\right)$ is a continuous operator $\mathcal{S}\left(\mathbb{R}^{n}\right) \longrightarrow \mathcal{S}\left(\mathbb{R}^{n}\right)$ and can hence be extended into a continuous operator $\mathcal{S}^{\prime}\left(\mathbb{R}^{n}\right) \longrightarrow \mathcal{S}^{\prime}\left(\mathbb{R}^{n}\right)$. It follows by duality that if $\widehat{A} \in$ $G_{\rho}^{m}\left(\mathbb{R}^{n}\right)$, then $\widehat{A}^{*} \in G_{\rho}^{m}\left(\mathbb{R}^{n}\right)$ (cf. [28], Theorem 23.5).

One also shows that $\left(\left[28\right.\right.$, Theorem 23.6]) if $\widehat{A} \in G_{\rho}^{m}\left(\mathbb{R}^{n}\right)$ and $\widehat{B} \in G_{\rho}^{m^{\prime}}\left(\mathbb{R}^{n}\right)$, then $\widehat{C}=\widehat{A} \widehat{B} \in G_{\rho}^{m+m^{\prime}}\left(\mathbb{R}^{n}\right)$.

\section{WEYL VERSUS BORN-JORDAN SYMBOL}

4.1. General results. Comparing the expressions (2.4) and (2.14) giving the harmonic representations of, respectively, Weyl and Born-Jordan operators one sees that if $\widehat{A}=\mathrm{Op}_{\mathrm{W}}(a)=\mathrm{Op}_{\mathrm{BJ}}(b)$, then the symbols $a$ and $b$ are related by the convolution relation $b * \theta_{\mathrm{BJ}}=a$; equivalently, taking the (symplectic) Fourier transform of each side,

$$
a_{\sigma}(z)=b_{\sigma}(z) \operatorname{sinc}\left(\frac{p x}{2 \hbar}\right) .
$$

The difficulty in recovering $b_{\sigma}$ from $a_{\sigma}$ comes from the fact that the sinc function has infinitely many zeros; in fact $\operatorname{sinc}(p x / 2 \hbar)=0$ for all points $z=(x, p)$ such that $p x=2 N \pi \hbar$ for a non-zero integer $N$. We are thus confronted with a division problem. Notice in addition that if the solution $b$ exists, then it is not unique: assume that $c(z)=e^{-i \sigma\left(z, z_{0}\right) / \hbar}$, where $p_{0} x_{0}=2 N \pi \hbar(N \in \mathbb{Z}, N \neq 0)$. We have $c_{\sigma}(z)=(2 \pi \hbar)^{n} \delta\left(z-z_{0}\right)$ and hence by (2.14)

$$
\mathrm{Op}_{\mathrm{BJ}}(c)=\int \delta\left(z-z_{0}\right) \operatorname{sinc}\left(\frac{p x}{2 \hbar}\right) \widehat{T}(z) d^{2 n} z=0 .
$$

It follows that if $\mathrm{Op}_{\mathrm{BJ}}(b)=\mathrm{Op}_{\mathrm{W}}(a)$, then we also have $\mathrm{Op}_{\mathrm{BJ}}(b+c)=\mathrm{Op}_{\mathrm{W}}(a)$. Now, in [9, Theorem 7] we have proven that equation (4.1) always has a (nonunique) solution in $b \in \mathcal{S}\left(\mathbb{R}^{2 n}\right)$ for every given $a \in \mathcal{S}\left(\mathbb{R}^{2 n}\right)$; our proof used the theory of division of distributions. Thus every Weyl operator has a Born-Jordan symbol; equivalently,

Proposition 4.1. For every continuous linear operator $\widehat{A}: \mathcal{S}\left(\mathbb{R}^{n}\right) \longrightarrow \mathcal{S}^{\prime}\left(\mathbb{R}^{n}\right)$ there exists $b \in \mathcal{S}^{\prime}\left(\mathbb{R}^{2 n}\right)$ such that $\widehat{A}=\mathrm{Op}_{\mathrm{BJ}}(b)$.

Notice that the existence of the solution $b$ of (4.1), as established in [9], is a purely qualitative result; it does not tell us anything about the properties of that solution. 
4.2. Weyl symbol of a Born-Jordan operator. We are going to show that every Born-Jordan operator with symbol in one of the Shubin classes $\Gamma_{\rho}^{m}\left(\mathbb{R}^{2 n}\right)$ is a Weyl operator with symbol in the same symbol class and produce an asymptotic expansion for the latter. For this we will need the following elementary inequalities (see for instance Chazarain and Piriou [6] or Hörmander [24]).

Lemma 4.2. Let $\xi$ and $\eta$ be positive numbers and $m \in \mathbb{R}$. We have

$$
\min \left\{\xi^{m}, \eta^{m}\right\} \leq C(\xi+\eta)^{m},
$$

where $C=\max \left\{1,2^{-m}\right\}$ and

$$
\left(1+|\xi-\eta|^{2}\right)^{m} \leq 2^{|m|}\left(1+|\xi|^{2}\right)^{m}\left(1+|\eta|^{2}\right)^{|m|} .
$$

The estimate (4.3) is usually referred to as Peetre's inequality in the literature on pseudodifferential operators.

Theorem 4.3. Let $\widehat{A}_{\mathrm{BJ}}=\mathrm{Op}_{\mathrm{BJ}}(a)$ with symbol $a \in \Gamma_{\rho}^{m}\left(\mathbb{R}^{2 n}\right)$.

(i) For every $\tau \in \mathbb{R}$ there exists $a_{\tau} \in \Gamma_{\rho}^{m}\left(\mathbb{R}^{2 n}\right)$ such that $\widehat{A}_{\mathrm{BJ}}=\mathrm{Op}_{\tau}\left(a_{\tau}\right)$. Here $a_{\tau}$ has the following asymptotic expansion:

$$
a_{\tau}(x, p) \sim \sum_{\alpha \in \mathbb{N}^{n}} \frac{(i \hbar)^{|\alpha|}\left(\tau^{|\alpha|+1}-(\tau-1)^{|\alpha|+1}\right)}{\alpha !(|\alpha|+1)} \partial_{x}^{\alpha} \partial_{p}^{\alpha} a(x, p) .
$$

(ii) In particular $\widehat{A}_{\mathrm{BJ}}=\mathrm{Op}_{\mathrm{BJ}}\left(\right.$ a) is a Weyl operator $\widehat{A}_{\mathrm{W}}=\mathrm{Op}_{\mathrm{W}}\left(a_{\mathrm{W}}\right)$ with symbol $a_{\mathrm{W}} \in \Gamma_{\rho}^{m}\left(\mathbb{R}^{2 n}\right)$, having the asymptotic expansion

$$
a_{\mathrm{W}}(x, p) \sim \sum_{\substack{\alpha \in \mathbb{N}^{n} \\|\alpha| \text { even }}} \frac{1}{\alpha !(|\alpha|+1)}\left(\frac{i \hbar}{2}\right)^{|\alpha|} \partial_{x}^{\alpha} \partial_{p}^{\alpha} a(x, p)
$$

and we have $a_{\mathrm{W}}-a \in \Gamma_{\rho}^{m-2 \rho}\left(\mathbb{R}^{2 n}\right)$.

Proof. Property (ii) follows from (i) choosing $\tau=\frac{1}{2}$.

(i) Consider the $\tau$-pseudodifferential operator $\widehat{A}_{\tau}=\mathrm{Op}_{\tau}(a)$ :

$$
\widehat{A}_{\tau} \psi(x)=\left(\frac{1}{2 \pi \hbar}\right)^{n} \int e^{\frac{i}{\hbar} p(x-y)} a((1-\tau) x+\tau y, p) \psi(y) d^{n} y d^{n} p
$$

and set

$$
a_{\mathrm{BJ}}(x, y, p)=\int_{0}^{1} a((1-\tau) x+\tau y, p) d \tau .
$$

We thus have, using (2.11),

$$
\widehat{A}_{\mathrm{BJ}} \psi(x)=\left(\frac{1}{2 \pi \hbar}\right)^{n} \int e^{\frac{i}{\hbar} p(x-y)} a_{\mathrm{BJ}}(x, y, p) \psi(y) d^{n} y d^{n} p
$$

which is of the type (3.5). Let us show that $a_{\mathrm{BJ}} \in \Pi_{\rho}^{m}\left(\mathbb{R}^{3 n}\right)$, i.e., that we have estimates of the type

$$
\left|\partial_{x}^{\alpha} \partial_{y}^{\beta} \partial_{p}^{\gamma} a_{\mathrm{BJ}}(x, y, p)\right| \leq C_{\alpha, \beta, \gamma}\langle(x, y, p)\rangle^{m-\rho|\alpha+\beta+\gamma|}\langle x-y\rangle^{m^{\prime}+\rho|\alpha+\beta+\gamma|}
$$

for some $m^{\prime} \in \mathbb{R}$ independent of $\alpha, \beta, \gamma$. The result will follow using Proposition 3.6. Let us set

we have

$$
b_{\tau}(x, y, p)=a((1-\tau) x+\tau y, p)
$$

$$
\partial_{x}^{\alpha} \partial_{y}^{\beta} \partial_{p}^{\gamma} b_{\tau}(x, y, p)=(1-\tau)^{|\alpha|} \tau^{|\beta|}\left(\partial_{x}^{\alpha+\beta} \partial_{p}^{\gamma} a\right)((1-\tau) x+\tau y, p) .
$$


Hence, since $a \in \Gamma_{\rho}^{m}\left(\mathbb{R}^{n}\right)$, we have by (3.1) the estimates

$$
\left|\partial_{x}^{\alpha} \partial_{y}^{\beta} \partial_{p}^{\gamma} b_{\tau}(x, y, p)\right| \leq C_{\alpha+\beta, \gamma}(1-\tau)^{|\alpha|} \tau^{|\beta|}\langle((1-\tau) x+\tau y, p)\rangle^{m-\rho|\alpha+\beta+\gamma|} .
$$

Now, by Peetre's inequality (4.3) there exists a constant $C=C(m, \rho, \alpha, \beta, \gamma)>0$ such that the estimates

$$
\langle((1-\tau) x+\tau y, p)\rangle^{m-\rho|\alpha+\beta+\gamma|} \leq C\langle(x, p)\rangle^{m-\rho|\alpha+\beta+\gamma|}\langle\tau(x-y)\rangle^{|m|+\rho|\alpha+\beta+\gamma|}
$$

and

$$
\langle((1-\tau) x+\tau y, p)\rangle^{m-\rho|\alpha+\beta+\gamma|} \leq C\langle(y, p)\rangle^{m-\rho|\alpha+\beta+\gamma|}\langle(1-\tau)(x-y)\rangle^{|m|+\rho|\alpha+\beta+\gamma|}
$$

hold, and hence

$$
\begin{aligned}
& \langle((1-\tau) x+\tau y, p)\rangle^{m-\rho|\alpha+\beta+\gamma|} \\
& \quad \leq C \min \left\{\langle(x, p)\rangle^{m-\rho|\alpha+\beta+\gamma|},\langle(y, p)\rangle^{m-\rho|\alpha+\beta+\gamma|}\right\}\langle x-y\rangle^{|m|+\rho|\alpha+\beta+\gamma|} .
\end{aligned}
$$

This implies, using the inequality (4.2), that

$$
\langle((1-\tau) x+\tau y, p)\rangle^{m-\rho|\alpha+\beta+\gamma|} \leq C^{\prime}\langle(x, y, p)\rangle^{m-\rho|\alpha+\beta+\gamma|}\langle x-y\rangle^{|m|+\rho|\alpha+\beta+\gamma|} .
$$

Together with (4.8) this inequality implies (4.7) with $m^{\prime}=|m|$ after an integration on $\tau$. The asymptotic expansion (4.4) follows by using the expansion of the $\tau$ symbol $a_{\tau}$ in (3.8), in terms of the amplitude $b(x, y, p)=a_{\mathrm{BJ}}(x, y, p)$ in (4.6). Namely, observe that

$$
\begin{aligned}
\partial_{p}^{\beta+\gamma}\left(i \hbar \partial_{x}\right)^{\beta}\left(-i \hbar \partial_{y}\right)^{\gamma} & \left.b(x, y, p)\right|_{y=x} \\
= & (i \hbar)^{|\beta+\gamma|}(-1)^{|\gamma|} \int_{0}^{1}(1-t)^{|\beta|} t^{|\gamma|} \partial_{p}^{\beta+\gamma} \partial_{x}^{\beta+\gamma} a(x, p) d t \\
= & (i \hbar)^{|\beta+\gamma|}(-1)^{|\gamma|} \partial_{p}^{\beta+\gamma} \partial_{x}^{\beta+\gamma} a(x, p) \int_{0}^{1}(1-t)^{|\beta|} t^{|\gamma|} d t .
\end{aligned}
$$

Setting $\alpha=\beta+\gamma$, so that $\beta=\alpha-\gamma$, we have

$$
\frac{1}{\beta ! \gamma !}=\left(\begin{array}{l}
\alpha \\
\gamma
\end{array}\right) \frac{1}{\alpha !}
$$

and hence

$$
\begin{aligned}
\sum_{\gamma \leq \alpha}\left(\begin{array}{l}
\alpha \\
\gamma
\end{array}\right)[\tau(1-t)]^{|\alpha-\gamma|}(-1)^{|\gamma|} & {[(1-\tau) t]^{|\gamma|} } \\
& =\prod_{j=1}^{n} \sum_{\gamma_{j} \leq \alpha_{j}}\left(\begin{array}{c}
\alpha_{j} \\
\gamma_{j}
\end{array}\right)[\tau(1-t)]^{\left|\alpha_{j}-\gamma_{j}\right|}[-(1-\tau) t]^{\left|\gamma_{j}\right|} \\
& =\prod_{j=1}^{n}[\tau(1-t)-(1-\tau) t]^{\alpha_{j}} \\
& =(\tau-t)^{|\alpha|}
\end{aligned}
$$

Computing the integral

$$
\int_{0}^{1}(\tau-t)^{|\alpha|} d t=\frac{\tau^{|\alpha|+1}-(\tau-1)^{|\alpha|+1}}{|\alpha|+1}
$$

we immediately obtain the asymptotic expansion for $a_{\tau}(x, p)$ in (4.4). This concludes the proof. 
Notice that the asymptotic formula (4.5) yields exact results when the BornJordan symbol $a$ is a polynomial in the variables $x_{j}, p_{k}$. For instance, when $n=1$ and $a(z)=a_{r s}(z)=x^{r} p^{s}$ it leads to

$$
a_{r s, \mathrm{~W}}(x, p)=\sum_{\substack{k \leq \inf (r, s) \\
k \text { even }}}\left(\frac{i \hbar}{2}\right)^{k} \frac{k !}{k+1}\left(\begin{array}{l}
r \\
k
\end{array}\right)\left(\begin{array}{l}
s \\
k
\end{array}\right) x^{r-k} p^{s-k} .
$$

We refer to Domingo and Galapon [22] for a general discussion of quantization of monomials.

Using [28, Definition 23.4, the result above has the following interesting consequence.

Corollary 4.4. A Born-Jordan operator $\widehat{A}_{\mathrm{BJ}}=\mathrm{Op}_{\mathrm{BJ}}(a)$ with symbol $a \in \Gamma_{\rho}^{m}\left(\mathbb{R}^{2 n}\right)$ belongs to $G_{\rho}^{m}\left(\mathbb{R}^{n}\right)$.

In many cases this result reduces the study of Born-Jordan operators to that of Shubin operators.

4.3. The Born-Jordan symbol of a Weyl operator. We now address the more difficult problem of finding the Born-Jordan symbol of a given Weyl operator in $G_{\rho}^{m}\left(\mathbb{R}^{2 n}\right)$. As already observed the analysis in [9] did not provide an explicit formula for it because of division problems. It is remarkable that, nevertheless, an explicit and general asymptotic expansion can be written down when the symbol belongs to one of the classes $\Gamma_{\rho}^{m}\left(\mathbb{R}^{2 n}\right)$. To this end we need a preliminary lemma about the formal power series arising in (4.5).

Lemma 4.5. Consider the power series

$$
\sum_{\substack{\alpha \in \mathbb{N}^{n} \\|\alpha| \text { even }}} \frac{1}{\alpha !(|\alpha|+1)} x^{\alpha}
$$

Its formal reciprocal is given by the series $\sum_{\alpha \in \mathbb{N}^{n}} \frac{c_{\alpha}}{\alpha !} x^{\alpha}$, where $c_{0}=1$ and, for $\alpha \neq 0$,

$$
c_{\alpha}=\alpha ! \sum_{j=1}^{|\alpha|}(-1)^{j} \sum_{\substack{\alpha^{(1)}+\ldots+\alpha^{(j)}=\alpha \\\left|\alpha^{(1)}\right|, \ldots,\left|\alpha^{(j)}\right| \neq 0 \text { even }}} \frac{1}{\alpha^{(1)} ! \cdots \alpha^{(j) !} !\left(\alpha^{(1)} \mid+1\right) \cdots\left(\left|\alpha^{(j)}\right|+1\right)} .
$$

Proof. The proof is straightforward: we expand

$$
\left(1+\sum_{|\alpha| \neq 0 \text { even }} \frac{1}{\alpha !(|\alpha|+1)} x^{\alpha}\right)^{-1}
$$

as a geometric series and collect the similar terms. Alternatively, we could also apply the Faà di Bruno formula generalizing the chain rule to the derivatives at $x=0$ of the function $x \longmapsto g(f(x))$, where $g(t)=1 /(1+t)$ and $f(x)=$

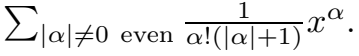

Remark 4.6. The series $\sum_{\alpha \in \mathbb{N}^{n}} \frac{c_{\alpha}}{\alpha !} x^{\alpha}$ in the above lemma has a positive but finite radius of convergence. For example in dimension $n=1$ the radius is $\pi$, because the (complex) zeros of the function $\sinh x / x$ closest to 0 are $\pm i \pi$ (see the computations after the proof of Theorem 4.7 below). However, the above result suffices for the 
following applications, where we will work in the framework of formal power series, as usual in the pseudodifferential calculus.

Let us now prove our second main result.

Theorem 4.7. Consider a Weyl operator $\widehat{A}_{\mathrm{W}}=\mathrm{Op}_{\mathrm{W}}(a)$ with Weyl symbol $a \in$ $\Gamma_{\rho}^{m}\left(\mathbb{R}^{2 n}\right)$. Let $b \in \Gamma_{\rho}^{m}\left(\mathbb{R}^{2 n}\right.$ ) be any symbol (whose existence is guaranteed by Proposition (3.4) with the following asymptotic expansion:

$$
b(x, p) \sim \sum_{\substack{\alpha \in \mathbb{N}^{n} \\|\alpha| \text { even }}} \frac{c_{\alpha}}{\alpha !}\left(\frac{i \hbar}{2}\right)^{|\alpha|} \partial_{x}^{\alpha} \partial_{p}^{\alpha} a(x, p),
$$

where the coefficients $c_{\alpha}$ are given in (4.10) $\left(c_{0}=1\right)$.

Let $\widehat{A}_{\mathrm{BJ}}=\mathrm{Op}_{\mathrm{BJ}}(b)$ be the corresponding Born-Jordan operator. Then

$$
\widehat{A}_{\mathrm{BJ}}=\widehat{A}_{\mathrm{W}}+R,
$$

where $R$ is a pseudodifferential operator with symbol in the Schwartz space $\mathcal{S}\left(\mathbb{R}^{2 n}\right)$.

Proof. The operator $\widehat{A}_{\mathrm{BJ}}=\mathrm{Op}_{\mathrm{BJ}}(b)$ by Theorem 4.3 can be written as a Weyl operator with Weyl symbol

$$
a_{\mathrm{W}}(x, p) \sim \sum_{\substack{\alpha \in \mathbb{N}^{n} \\|\alpha| \text { even }}} \frac{1}{\alpha !(|\alpha|+1)}\left(\frac{i \hbar}{2}\right)^{|\alpha|} \partial_{x}^{\alpha} \partial_{p}^{\alpha} b(x, p) .
$$

Now we substitute in this expression the asymptotic expansion (4.11) for $b$ and we use the fact that the formal differential operators given by the series

$$
\sum_{\substack{\alpha \in \mathbb{N}^{n} \\|\alpha| \text { even }}} \frac{1}{\alpha !(|\alpha|+1)}\left(\frac{i \hbar}{2}\right)^{|\alpha|} \partial_{x}^{\alpha} \partial_{p}^{\alpha} \text { and } \sum_{\substack{\alpha \in \mathbb{N}^{n} \\|\alpha| \text { even }}} \frac{c_{\alpha}}{\alpha !}\left(\frac{i \hbar}{2}\right)^{|\alpha|} \partial_{x}^{\alpha} \partial_{p}^{\alpha}
$$

are inverses of each other in view of Lemma 4.5 (to see this, formally replace $x=\left(x_{1}, \ldots, x_{n}\right)$ in Lemma 4.5 by $\left.(i \hbar / 2)\left(\partial_{x_{1}} \partial_{p_{1}}, \ldots, \partial_{x_{n}} \partial_{p_{n}}\right)\right)$. It follows that

$$
a_{\mathrm{W}}-a \in \bigcap_{N \in \mathbb{N}} \Gamma_{\rho}^{-N}\left(\mathbb{R}^{2 n}\right)=\mathcal{S}\left(\mathbb{R}^{2 n}\right)
$$

and hence (4.12).

Notice that in dimension $n=1$ we have

$$
\sum_{k \geq 0 \text { even }} \frac{1}{k !(k+1)} x^{k}=\frac{1}{x} \sum_{k \geq 0 \text { even }} \frac{1}{(k+1) !} x^{k+1}=\frac{\sinh x}{x}=: F(x)
$$

so that $c_{k}=\left.\partial_{x}^{k}(1 / F(x))\right|_{x=0}$. In particular, $c_{k}=0$ for odd $k$. In this case the series expansion of $1 / F(x)$ is particularly easy, since it coincides with the MacLaurin series expansion of the function

$$
\frac{1}{F(x)}=x \operatorname{cosech} x=\sum_{k \geq 0 \text { even }} \frac{2-2^{k}}{k !} B_{k} x^{k},
$$

where the $B_{k}$ are the Bernoulli numbers

$$
B_{k}=\lim _{x \rightarrow 0} f^{(k)}(x),
$$


with

$$
f(x)=\frac{x}{e^{x}-1}
$$

More explicitly,

$$
\frac{1}{F(x)}=x \operatorname{cosech} x=1-\frac{1}{6} x^{2}+\frac{7}{360} x^{4}-\frac{31}{15120} x^{6}+\frac{127}{604800} x^{8}-\cdots
$$

and the coefficients $c_{k}$, with $k$ even, are provided by

$$
c_{0}=1, c_{2}=-\frac{1}{3}, c_{4}=\frac{7}{15}, c_{6}=-\frac{31}{21}, c_{8}=\frac{127}{15}, \ldots \quad(n=1) .
$$

In this case formula (4.11) takes the simple form

$$
b(x, p) \sim \sum_{k \geq 0 \text { even }} \frac{c_{k}}{k !}\left(\frac{i \hbar}{2}\right)^{k} \partial_{x}^{k} \partial_{p}^{k} a(x, p) .
$$

As in the case of formula (4.9), the asymptotic expansion (4.11) becomes exact (and reduces to a finite sum) when the symbol $a$ is a polynomial. For instance, assuming $n=1$ choose $a(z)=a_{r s}(z)=x^{r} p^{s}$. Then the formula above yields

$$
\begin{aligned}
b_{r s, \mathrm{BJ}}(x, p) & =\sum_{k \leq \inf (r, s), k \text { even }} k ! c_{k}\left(\frac{i \hbar}{2}\right)^{k}\left(\begin{array}{l}
r \\
k
\end{array}\right)\left(\begin{array}{l}
s \\
k
\end{array}\right) x^{r-k} p^{s-k} . \\
& =\sum_{k \leq \inf (r, s), k \text { even }} k !\left(2-2^{k}\right) B_{k}\left(\frac{i \hbar}{2}\right)^{k}\left(\begin{array}{l}
r \\
k
\end{array}\right)\left(\begin{array}{l}
s \\
k
\end{array}\right) x^{r-k} p^{s-k},
\end{aligned}
$$

where the $B_{k}$ are the Bernoulli numbers defined in (4.15).

We also make the following remark: formulas (4.13) and (4.14) show that (modulo a term in $\mathcal{S}\left(\mathbb{R}^{2 n}\right)$ ) a Weyl operator with symbol in $\Gamma_{\rho}^{m}\left(\mathbb{R}^{2 n}\right)$ has a Born-Jordan symbol belonging to the same class $\Gamma_{\rho}^{m}\left(\mathbb{R}^{2 n}\right)$. This is however by no means a uniqueness result since, as we have already observed, we have $\mathrm{Op}_{\mathrm{BJ}}(b+c)=0$ for all symbols $c(z)=e^{-i \sigma\left(z, z_{0}\right) / \hbar}$, where $p_{0} x_{0}=2 N \pi \hbar(N \in \mathbb{Z}, N \neq 0)$. Observe that such a symbol $c$ belongs to none of the symbol classes $\Gamma_{\rho}^{m}\left(\mathbb{R}^{2 n}\right)$.

\section{Regularity AND GLOBAL HYPOELLIPTICITY RESUltS}

In order to define the Sobolev-Shubin spaces (cf. 28, Definition 25.3), we recall the definition of anti-Wick operators. The anti-Wick operator $\mathrm{Op}_{\mathrm{AW}}(a)$ with symbol $a$ is defined by

$$
\mathrm{Op}_{\mathrm{AW}}(a) f=\int a(z) P_{z} f d^{2 n} z
$$

where $P_{z} f(t)=\left\langle f, \Phi_{z}\right\rangle \Phi_{z}(t)$ are orthogonal projections on $L^{2}\left(\mathbb{R}^{n}\right)$ on the functions $\Phi_{z}(t)=\pi^{-n / 4} e^{i t p} e^{-\frac{|t-x|^{2}}{2}}$ (i.e., phase-space shifts of the Gaussian $\pi^{-n / 4} e^{-\frac{|t|^{2}}{2}}$ ).

Definition 5.1. For $s \in \mathbb{R}$ consider the anti-Wick symbol $\langle z\rangle^{s}, z \in \mathbb{R}^{2 n}$, and let $A_{s}=\mathrm{Op}_{\mathrm{AW}}(a)$ be the corresponding anti-Wick operator. The Sobolev-Shubin space $Q^{s}$ is defined by

$$
Q^{s}=\left\{f \in S^{\prime}\left(\mathbb{R}^{n}\right): A_{s} f \in L^{2}\left(\mathbb{R}^{n}\right)\right\}=A_{s}^{-1} L^{2}\left(\mathbb{R}^{n}\right) .
$$


Our reduction result Theorem 4.3 allows us to transpose to Born-Jordan operators all known continuity results for Weyl operators with symbol in the symbol classes $\Gamma_{\rho}^{m}\left(\mathbb{R}^{2 n}\right)$. For instance:

Proposition 5.2. Let $\widehat{A}_{\mathrm{BJ}}=\mathrm{Op}_{\mathrm{BJ}}(a)$ with symbol $a \in \Gamma_{\rho}^{m}\left(\mathbb{R}^{2 n}\right)$. We have

$$
\widehat{A}_{\mathrm{BJ}}: Q^{s}\left(\mathbb{R}^{n}\right) \rightarrow Q^{s-m}\left(\mathbb{R}^{n}\right) .
$$

In particular, if $m=s=0, \widehat{A}_{\mathrm{BJ}}$ is continuous on $L^{2}\left(\mathbb{R}^{n}\right)$.

Proof. By Corollary 4.4 the operator $\widehat{A}_{\mathrm{BJ}}$ is in the class $G_{\rho}^{m}\left(\mathbb{R}^{n}\right)$. The result follows by applying Theorem 25.2 in 28 .

It turns out that the Sobolev-Shubin spaces are particular cases of Feichtinger's modulation spaces [12, 13, 23; we do not discuss these here and refer to Cordero et al. 11] for a study of continuity properties of Born-Jordan operators in these spaces.

We next recall the notion of global hypoellipticity 28, 29, which plays an important role in the study of spectral theory for pseudodifferential operators (see the monograph [2] by Boggiatto et al.).

An operator $\widehat{A}: \mathcal{S}^{\prime}\left(\mathbb{R}^{n}\right) \longrightarrow \mathcal{S}^{\prime}\left(\mathbb{R}^{n}\right)$ which also maps $\mathcal{S}\left(\mathbb{R}^{n}\right)$ into itself is globally hypoelliptic if

$$
\psi \in \mathcal{S}^{\prime}\left(\mathbb{R}^{n}\right) \text { and } \widehat{A} \psi \in \mathcal{S}\left(\mathbb{R}^{n}\right) \Longrightarrow \psi \in \mathcal{S}\left(\mathbb{R}^{n}\right)
$$

(global hypoellipticity is thus not directly related to the usual notion of hypoellipticity [24, which is a local notion).

In 28] Shubin introduced the following subclass of $\Gamma_{\rho}^{m}\left(\mathbb{R}^{2 n}\right)$.

Definition 5.3. Let $m, m_{0} \in \mathbb{R}$ and $0<\rho \leq 1$. The symbol class $H \Gamma_{\rho}^{m, m_{0}}\left(\mathbb{R}^{2 n}\right)$ consists of all complex functions $a \in C^{\infty}\left(\mathbb{R}^{2 n}\right)$ such that

$$
C_{0}\langle z\rangle^{m_{0}} \leq|a(z)| \leq C_{1}\langle z\rangle^{m} \quad \text { for }|z|>R,
$$

for some $C_{0}, C_{1}, R>0$ and whose derivatives satisfy the following property: for every $\alpha \in \mathbb{N}^{2 n}$ there exists $C_{\alpha}>0$ such that

$$
\left|\partial_{z}^{\alpha} a(z)\right| \leq C_{\alpha}|a(z)|\langle z\rangle^{-\rho|\alpha|} \text { for }|z|>R .
$$

The symbol class $H \Gamma_{\rho}^{m, m_{0}}\left(\mathbb{R}^{2 n}\right)$ is insensitive to perturbations by lower order terms ([28], Lemma 25.1(c)):

Lemma 5.4. Let $a \in H \Gamma_{\rho}^{m, m_{0}}\left(\mathbb{R}^{2 n}\right)$ and $b \in \Gamma_{\rho}^{m^{\prime}}\left(\mathbb{R}^{2 n}\right)$. If $m^{\prime}<m_{0}$, then $a+b \in$ $H \Gamma_{\rho}^{m, m_{0}}\left(\mathbb{R}^{2 n}\right)$.

The interest of these symbol classes comes from the following property [28]: if $a \in H \Gamma_{\rho}^{m, m_{0}}\left(\mathbb{R}^{2 n}\right)$, then the Weyl operator $\widehat{A}=\mathrm{Op}_{\mathrm{W}}(a)$ is globally hypoelliptic. For instance, the Hermite operator $-\Delta+|x|^{2}$ is globally hypoelliptic since its Weyl symbol is $a(z)=|z|^{2}$, which is in $H \Gamma_{1}^{2,2}\left(\mathbb{R}^{2 n}\right)$. Moreover, if $\widehat{A}=\mathrm{Op}_{\mathrm{W}}(a)$ with $a \in H \Gamma_{\rho}^{m, m_{0}}\left(\mathbb{R}^{2 n}\right)$ the following stronger result holds:

$$
\psi \in \mathcal{S}^{\prime}\left(\mathbb{R}^{n}\right) \text { and } \widehat{A} \psi \in Q^{s}\left(\mathbb{R}^{n}\right) \Longrightarrow \psi \in Q^{s+m_{0}}\left(\mathbb{R}^{n}\right) .
$$


Proposition 5.5. Let $a \in H \Gamma_{\rho}^{m, m_{0}}\left(\mathbb{R}^{2 n}\right)$, with $m-2 \rho<m_{0}$.

(i) The Born-Jordan operator $\widehat{A}_{\mathrm{BJ}}=\mathrm{Op}_{\mathrm{BJ}}($ a) is globally hypoelliptic.

(ii) If $\psi$ is a tempered distribution such that $\widehat{A}_{\mathrm{BJ}} \psi \in Q^{s}\left(\mathbb{R}^{n}\right)$ for some $s \in \mathbb{R}$, then $\psi \in Q^{s+m_{0}}\left(\mathbb{R}^{n}\right)$.

Proof. In view of the discussion above it suffices to show that the Weyl symbol $a_{\mathrm{W}}$ of $\widehat{A}_{\mathrm{BJ}}$ belongs to the class $H \Gamma_{\rho}^{m, m_{0}}\left(\mathbb{R}^{2 n}\right)$. Now, by Theorem 4.3(ii) we have $a_{\mathrm{W}}-a \in \Gamma_{\rho}^{m-2 \rho}\left(\mathbb{R}^{2 n}\right)$, and hence the result using Lemma 5.4 .

\section{REFERENCES}

[1] P. Boggiatto, G. De Donno, and A. Oliaro, Time-frequency representations of Wigner type and pseudo-differential operators, Trans. Amer. Math. Soc. 362 (2010), no. 9, 4955-4981, DOI 10.1090/S0002-9947-10-05089-0. MR2645057

[2] Paolo Boggiatto, Ernesto Buzano, and Luigi Rodino, Global hypoellipticity and spectral theory, Mathematical Research, vol. 92, Akademie Verlag, Berlin, 1996. MR.1435282

[3] José Bonet, Leonhard Frerick, and Enrique Jordá, The division problem for tempered distributions of one variable, J. Funct. Anal. 262 (2012), no. 5, 2349-2358, DOI 10.1016/j.jfa.2011.12.006. MR2876408

[4] M. Born and P. Jordan, Zur Quantenmechanik, Z. Physik 34 (1925), 858-888.

[5] M. Born, W. Heisenberg, and P. Jordan, Zur Quantenmechanik II, Z. Physik 35 (1925), $557-615$.

[6] Jacques Chazarain and Alain Piriou, Introduction to the theory of linear partial differential equations, Studies in Mathematics and its Applications, vol. 14, North-Holland Publishing Co., Amsterdam-New York, 1982. Translated from the French: Introduction à la théorie des équations aux dérivées partielles linéaires, Gauthier-Villars, Paris, 1981. MR678605

[7] Leon Cohen, Generalized phase-space distribution functions, J. Mathematical Phys. 7 (1966), 781-786, DOI 10.1063/1.1931206. MR.0194105

[8] Leon Cohen, The Weyl operator and its generalization, Pseudo-Differential Operators. Theory and Applications, vol. 9, Birkhäuser/Springer Basel AG, Basel, 2013. MR3012699

[9] Elena Cordero, Maurice de Gosson, and Fabio Nicola, On the invertibility of BornJordan quantization, J. Math. Pures Appl. (9) 105 (2016), no. 4, 537-557, DOI 10.1016/j.matpur.2015.11.007. MR3471149

[10] E. Cordero, M. de Gosson, and F. Nicola, On the reduction of the interferences in the BornJordan distribution, Appl. Comput. Harmon. Anal., in press. arXiv:1601.03719 [math.FA] (2016)

[11] Elena Cordero, Maurice de Gosson, and Fabio Nicola, Time-frequency analysis of BornJordan pseudodifferential operators, J. Funct. Anal. 272 (2017), no. 2, 577-598, DOI 10.1016/j.jfa.2016.10.004. MR.3571901

[12] Hans G. Feichtinger, Un espace de Banach de distributions tempérées sur les groupes localement compacts abéliens, C. R. Acad. Sci. Paris Sér. A-B 290 (1980), no. 17, A791-A794. MR.580567

[13] Hans G. Feichtinger, On a new Segal algebra, Monatsh. Math. 92 (1981), no. 4, 269-289, DOI 10.1007/BF01320058. MR643206

[14] Maurice de Gosson, Symplectic geometry and quantum mechanics, Operator Theory: Advances and Applications, vol. 166, Birkhäuser Verlag, Basel, 2006. Advances in Partial Differential Equations (Basel). MR2241188

[15] Maurice A. de Gosson, Symplectic methods in harmonic analysis and in mathematical physics, Pseudo-Differential Operators. Theory and Applications, vol. 7, Birkhäuser/Springer Basel AG, Basel, 2011. MR2827662

[16] Maurice A. de Gosson, Symplectic covariance properties for Shubin and Born-Jordan pseudo-differential operators, Trans. Amer. Math. Soc. 365 (2013), no. 6, 3287-3307, DOI 10.1090/S0002-9947-2012-05742-4. MR.3034466

[17] Maurice A. de Gosson, Born-Jordan quantization and the equivalence of the Schrödinger and Heisenberg pictures, Found. Phys. 44 (2014), no. 10, 1096-1106, DOI 10.1007/s10701-0149831-z. MR3258594 
[18] Maurice A. de Gosson, Born-Jordan quantization: Theory and applications, Fundamental Theories of Physics, vol. 182, Springer, [Cham], 2016. MR3467497

[19] Maurice A. de Gosson, Born-Jordan quantization and the equivalence of the Schrödinger and Heisenberg pictures, Found. Phys. 44 (2014), no. 10, 1096-1106, DOI 10.1007/s10701-0149831-z. MR.3258594

[20] Maurice A. de Gosson, From Weyl to Born-Jordan quantization: The Schrödinger representation revisited, Phys. Rep. 623 (2016), 1-58, DOI 10.1016/j.physrep.2016.02.004. MR3475499

[21] Maurice de Gosson and Franz Luef, Preferred quantization rules: Born-Jordan versus Weyl. The pseudo-differential point of view, J. Pseudo-Differ. Oper. Appl. 2 (2011), no. 1, 115-139, DOI 10.1007/s11868-011-0025-6. MR2781145

[22] Herbert B. Domingo and Eric A. Galapon, Generalized Weyl transform for operator ordering: polynomial functions in phase space, J. Math. Phys. 56 (2015), no. 2, 022104, 13, DOI 10.1063/1.4907561. MR3390867

[23] Karlheinz Gröchenig, Foundations of time-frequency analysis, Applied and Numerical Harmonic Analysis, Birkhäuser Boston, Inc., Boston, MA, 2001. MR1843717

[24] Lars Hörmander, The analysis of linear partial differential operators. I: Distribution theory and Fourier analysis, 2nd ed., Grundlehren der Mathematischen Wissenschaften [Fundamental Principles of Mathematical Sciences], vol. 256, Springer-Verlag, Berlin, 1990. MR.1065993

[25] Michael Langenbruch, Real roots of polynomials and right inverses for partial differential operators in the space of tempered distributions, Proc. Roy. Soc. Edinburgh Sect. A 114 (1990), no. 3-4, 169-179, DOI 10.1017/S0308210500024367. MR.1055542

[26] Robert G. Littlejohn, The semiclassical evolution of wave packets, Phys. Rep. 138 (1986), no. 4-5, 193-291, DOI 10.1016/0370-1573(86)90103-1. MR845963

[27] N. H. McCoy, On the function in quantum mechanics which corresponds to a given function in classical mechanics, Proc. Natl. Acad. Sci. U.S.A. 18 (1932), no. 11, 674-676.

[28] M. A. Shubin, Pseudodifferential operators and spectral theory, Springer Series in Soviet Mathematics, Springer-Verlag, Berlin, 1987. Translated from the Russian by Stig I. Andersson. MR883081

[29] François Trèves, Introduction to pseudodifferential and Fourier integral operators. Vol. 1: Pseudodifferential operators, The University Series in Mathematics, Plenum Press, New YorkLondon, 1980. MR597144

[30] Ville Turunen, Born-Jordan time-frequency analysis, Harmonic analysis and nonlinear partial differential equations, RIMS Kôkyûroku Bessatsu, B56, Res. Inst. Math. Sci. (RIMS), Kyoto, 2016, pp. 107-186. MR 3617719

[31] H. Weyl, Quantenmechanik und Gruppentheorie, Zeitschrift für Physik 46 (1927).

Dipartimento di Matematica, Università di Torino, via Carlo Alberto 10, 10123 TORINO, ITALY

E-mail address: elena.cordero@unito.it

Faculty of Mathematics (NuHAG), University of Vienna, Oskar-Morgenstern-Platz 1, 1090 Vienna, Austria

E-mail address: maurice.de.gosson@univie.ac.at

Dipartimento di Scienze Matematiche, Politecnico di Torino, Corso Duca Degli Abruzzi 24, 10129 Torino, Italy

E-mail address: fabio.nicola@polito.it 